\title{
Autologous NK-cell-enrichment: preclinical setting phase, Shiraz experience
}

\section{CURRENT STATUS: POSTED}

Research Square

\section{Somayeh Rezaeifard}

Shiraz University of Medical Sciences

yuji Heike

st. luke's international hospital

Junichi Masuyama

NCO clinic

Alireza rezvani

Shiraz University of Medical Sciences

Reza vojdani

Shiraz University of Medical Sciences

Nasrollah Erfani

Shiraz University of Medical Sciences

verfanin@sums.ac.irCorresponding Author

ORCiD: https://orcid.org/0000-0002-4158-9128

DOI:

$10.21203 / \mathrm{rs} .2 .22044 / \mathrm{v} 1$

SUBJECT AREAS

Cell Communication and Signaling

KEYWORDS

NK cells, Cell therapy, Immune cell therapy, Breast cancer 
Abstract

Background: NK cell therapy has proven to be a promising approach for treatment of hematological malignancies and solid tumors. Masuyama et al. have recently introduced a new method for ex-vivo autologous NK cell expansion (Osaki method); resulting in the production of ample active NK cells for a promising cell therapy regimen. In order to start clinical trial phase I at Shiraz University of medical Sciences in collaboration with Masuyama clinic and St. Luck's International University Hospital, this preclinical setting study aimed to evaluate the proliferative efficacy of the method, the activation status of expanded autologous NK cells and the likely unwanted contamination of the final cell product.

Methods: PBMCs were isolated from $30 \mathrm{ml}$ of 5 healthy individuals' peripheral blood transferring directly to the specified initial culture bag containing antibodies for CD3, CD52 as well as IL-2 cytokine. The cells were cultured for 14-17 days in incubators; during which the cell received condition media, and underwent several passages into bigger culture bags. All the procedure was carried out in the clean room and associated facilities.

Results: Our results indicated that NK cells were expanded 510-fold in average (range 200-1100 fold), and the purity of NK cells per whole lymphocytes exceeded $68 \%$. The expanded cells were highly lytic as indicated by in-vitro cytotoxic assay; with strong expression of NKG2D and CD16. The prepared final cell products were negative for HCV, HBV, HIV, Mycoplasma and endotoxin.

Conclusion: In the preclinical setting phase, large numbers of activated and un-contaminated NK cells from $30 \mathrm{ml}$ of healthy individuals' peripheral blood were successfully generated. The method seems to provide ample clean cell product with no contamination; suitable to be infused back to the patients in phase I clinical trial.

\section{Full-text}

Due to technical limitations, full-text HTML conversion of this manuscript could not be completed. However, the manuscript can be downloaded and accessed as a PDF.

\section{Figures}
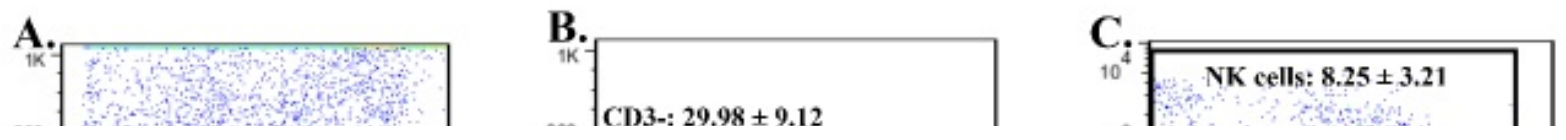


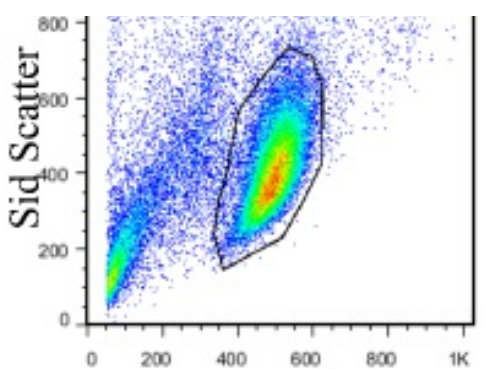

Forward Scatter
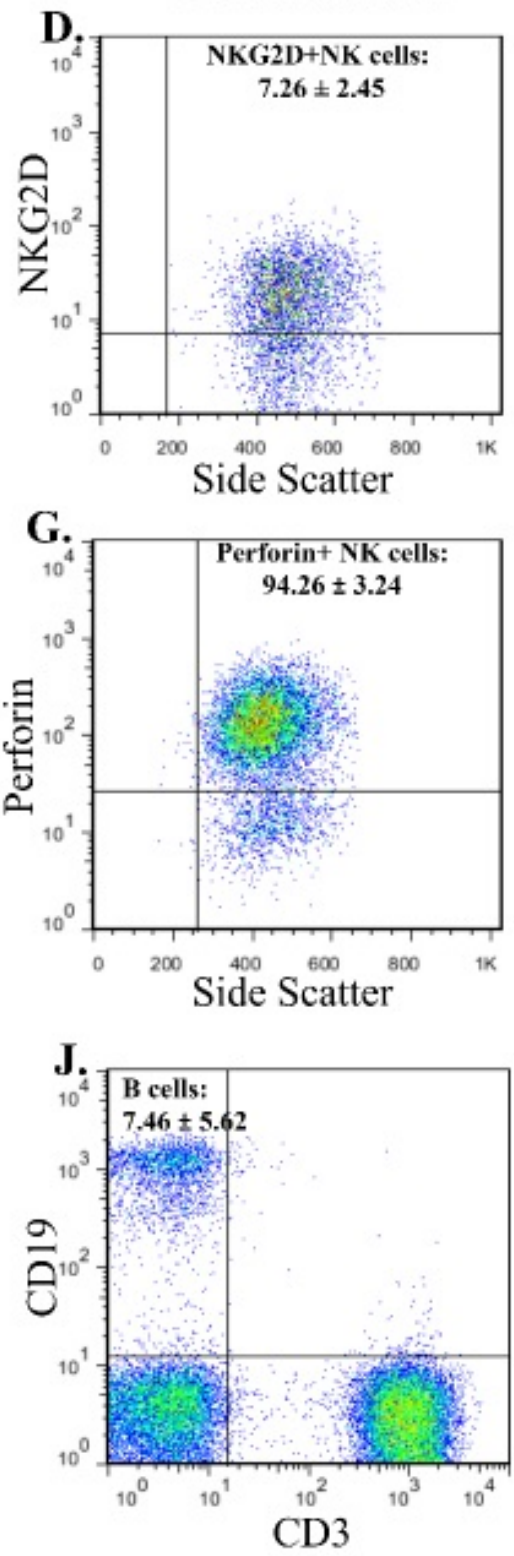
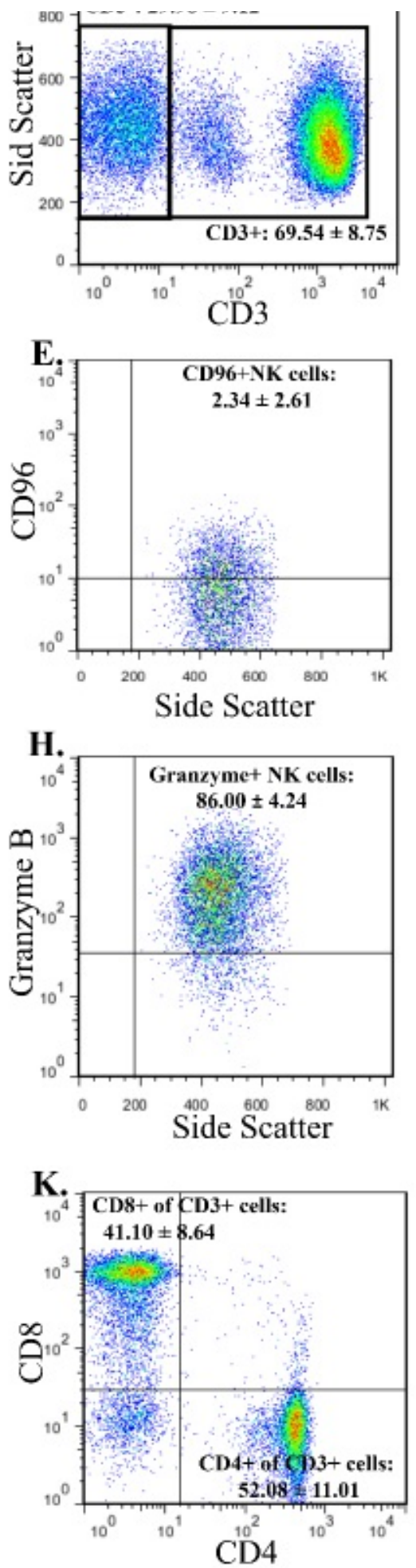
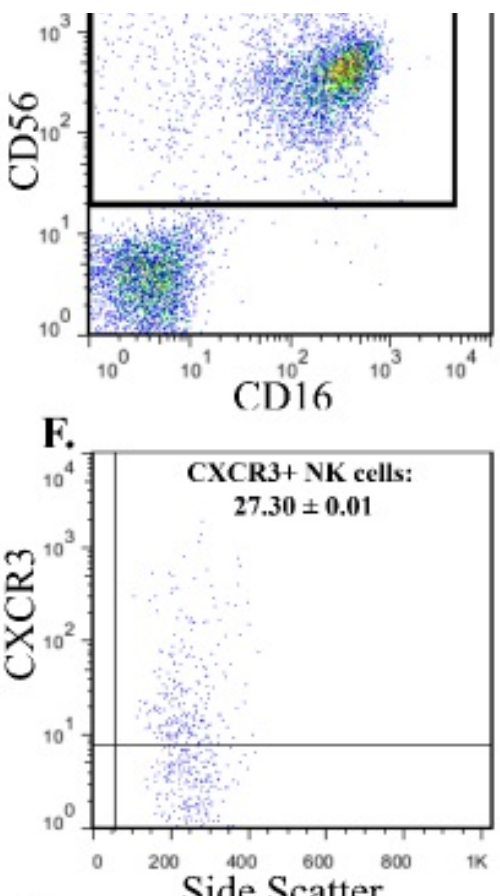

I.
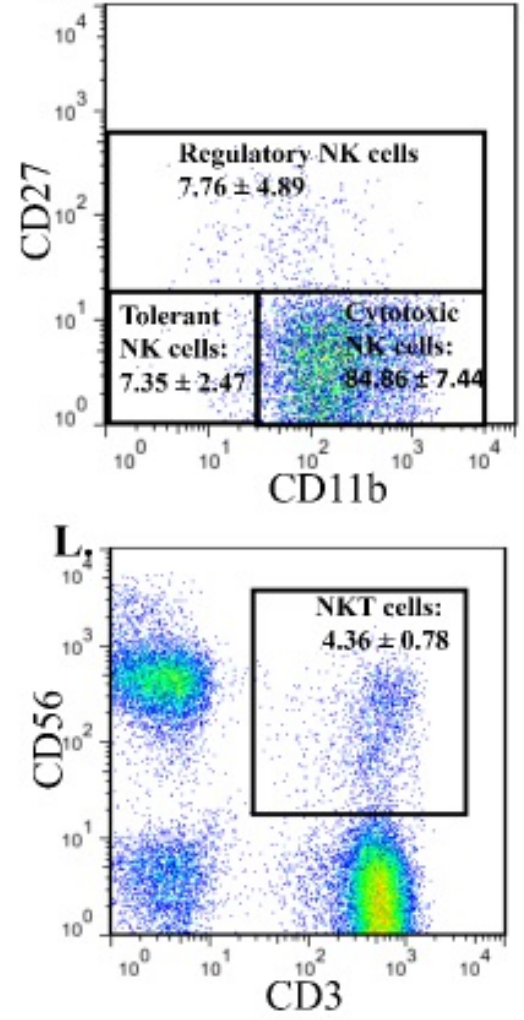

Figure 1

Flowcytometery analysis of the different immune cells before Osaki method of NK cells enrichment in healthy individuals A. Forward and Side Scatter dot plot and lymphocytes gate B. CD3- and C3+ cell population in lymphocyte gate C. NK cells in CD3- cell population D. NKG2D+ NK cells E. CD96+ NK cells F. CXCR3+ NK cells G. Perforin + NK cells H. 
Granzyme B+ NK cells I. Regulatory, Tolerant and cytotoxic subsets of NK cells J. B cells K.

$$
\text { CD4+ and CD8+ cells in CD3+ gate L. NKT cells }
$$
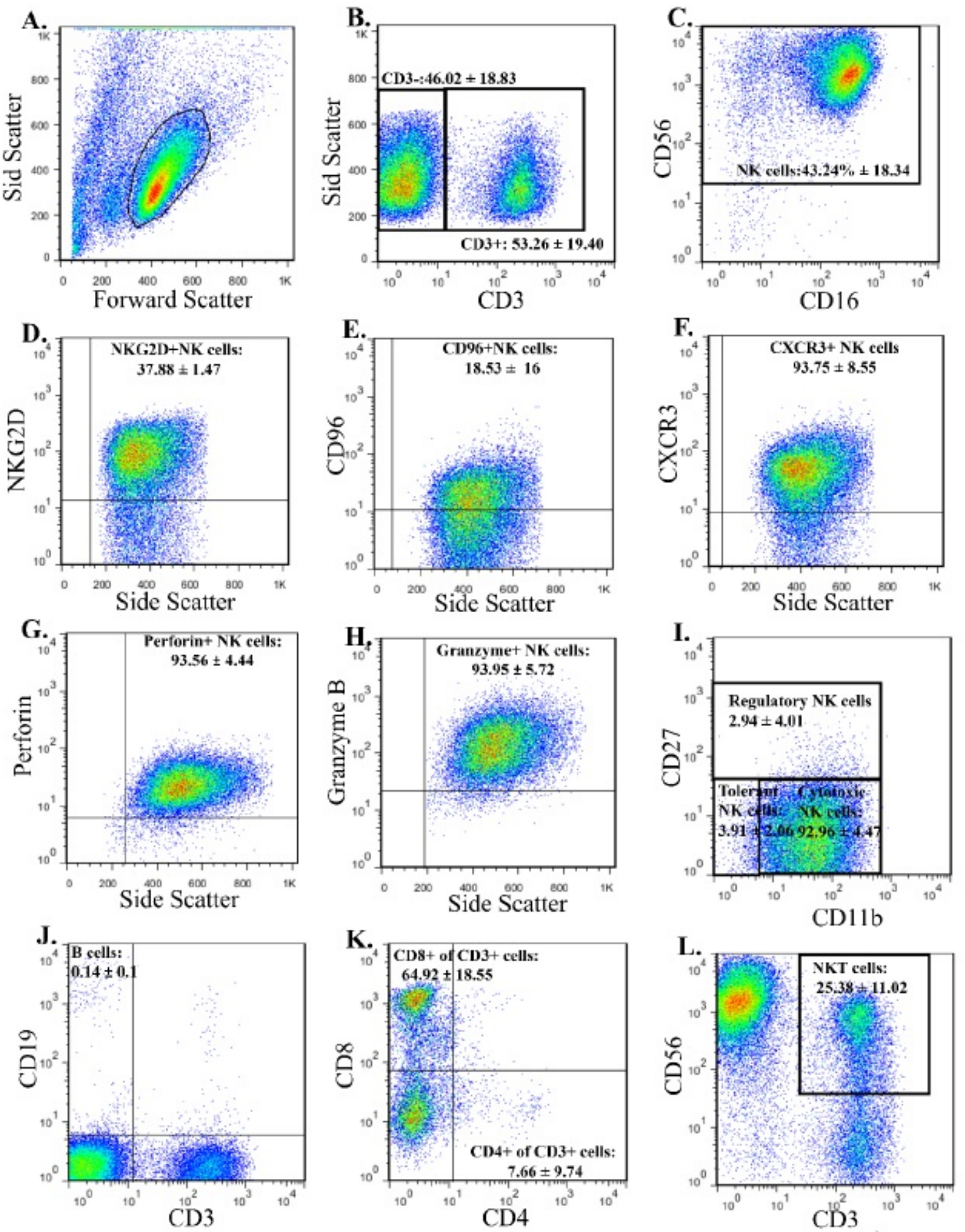

Figure 2

Flowcytometery analysis of the different immune cells after Osaki method of NK cells 
enrichment in healthy individuals A. Forward and Side Scatter dot plot and lymphocytes

gate B. CD3- and C3+ cell population in lymphocyte gate C. NK cells in CD3- cell population D. NKG2D+ NK cells E. CD96+ NK cells F. CXCR3+ NK cells G. Perforin+ NK cells H.

Granzyme B+ NK cells I. Regulatory, Tolerant and cytotoxic subsets of NK cells J. B cells K.

$$
\text { CD4+ and CD8+ cells in CD3+ gate L. NKT cells }
$$

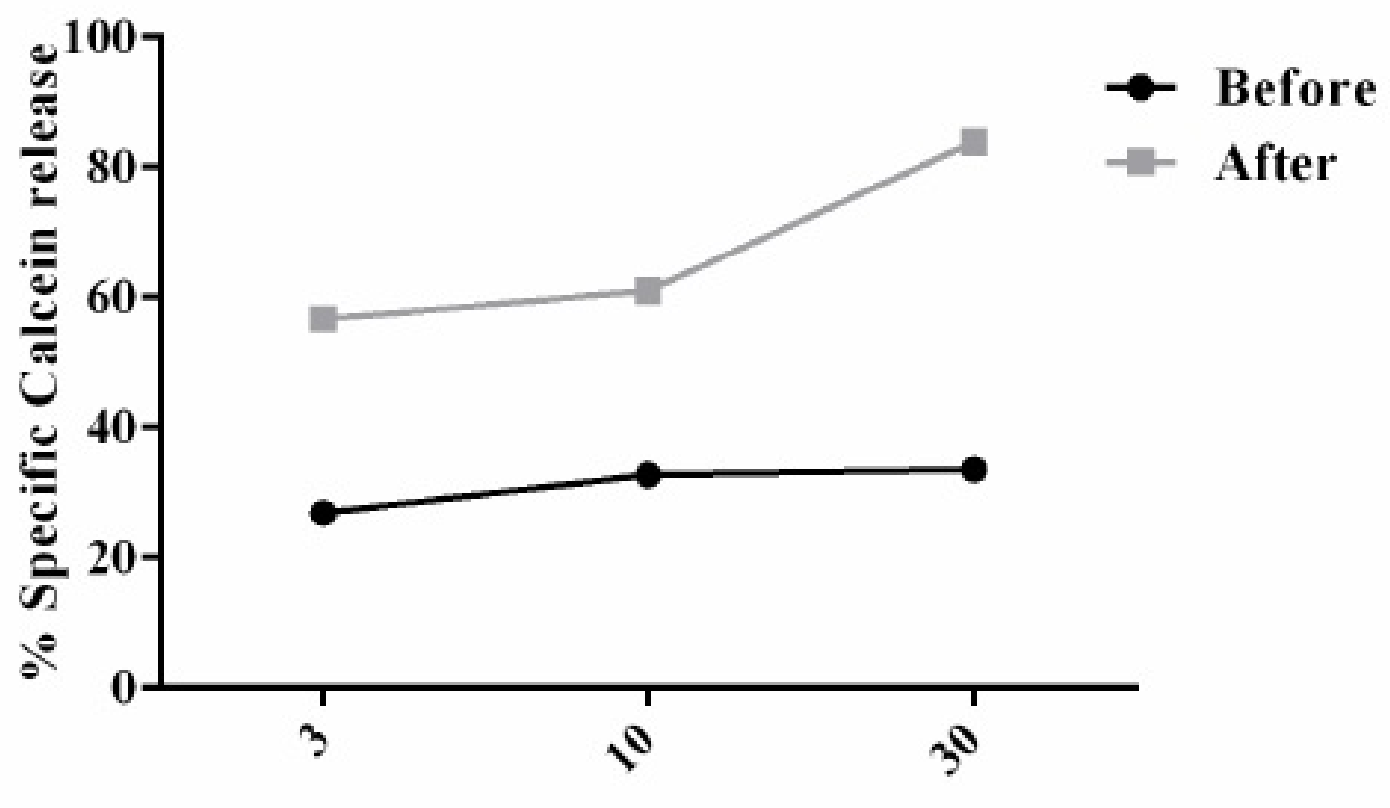

Effector/Target cells ratios

Figure 3

Calcein release killing assay before and after Osaki method of NK cells enrichment.. Effector (PBMCs)/ Target cells (K562 cells) ratios were 30, 10 and 3 
A.

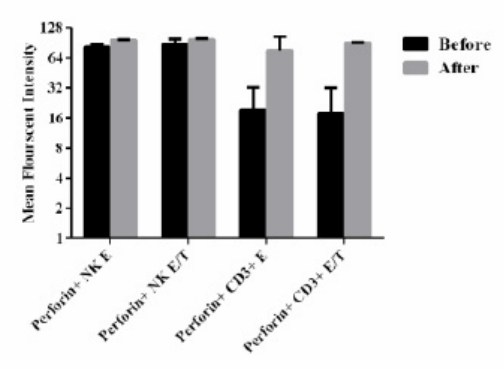

B.

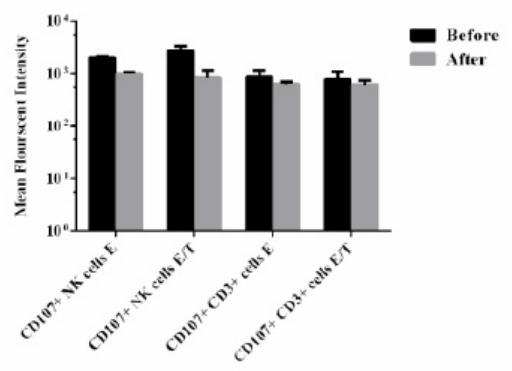

c.

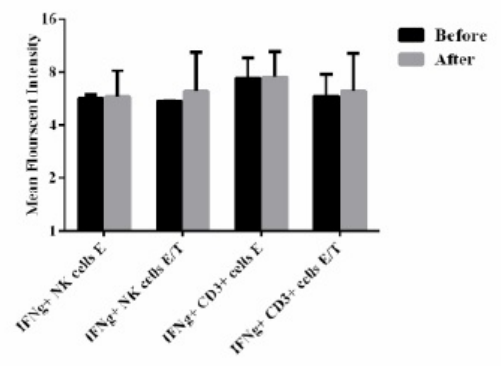

Figure 4

A. Lytic protein B. Degranulation C. IFN-Y production assay by flowcytometry in Effector and Effector/Target cells before and after Osaki method of NK cells enrichment. 